\title{
Clinical relevance of epidermal growth factor receptor (EGFR) alterations in human pancreatic tumors
}

\author{
ANTONIO LOZANO-LEON ${ }^{1}$, BEGONA VIEITES PEREZ-QUINTELA ${ }^{2}$, JULIO IGLESIAS-GARCIA ${ }^{1}$, \\ ADELA URISARRI-RUIZ ${ }^{3}$, JOSE LARIÑO-NOIA ${ }^{1}$, IHAB ABDULKADER $^{2}$, EVARISTO VARO $^{4}$, \\ JERONIMO FORTEZA ${ }^{2}$ and J. ENRIQUE DOMINGUEZ-MUÑOZ ${ }^{1}$
}

\author{
Departments of ${ }^{1}$ Gastroenterology and Foundation for Research in Digestive Diseases, ${ }^{2}$ Pediatric, \\ ${ }^{3}$ Pathology, and ${ }^{4}$ General Surgery, University Hospital Santiago de Compostela, Spain
}

Received November 5, 2010; Accepted December 30, 2010

DOI: $10.3892 /$ or.2011.1309

\begin{abstract}
Pancreatic cancer is a malignant neoplasm with an extremely poor prognosis. The mechanisms of aggressive growth and metastasis are currently not well understood. Expression of epidermal growth factor receptor (EGFR) has been suggested to be associated with the malignant transformation of pancreatic cancer. In this study, we examined the EGFR status of 52 pancreatic tumors by PCR-sequencing (exons 19 and 21), immunohistochemistry and FISH probes. We subsequently investigated the relationship between EGFR status and clinicopathological factors. Somatic alterations in EGFR (R841R, T571T and R831C) were observed only in ductal adenocarcinoma (3/34). In $4(8 \%)$ of the 52 tumors analyzed EGFR was overexpressed, $6(12 \%)$ of the tumors showed moderate expression while 19 (32\%) were weakly stained. EGFR overexpression (3+ score) was frequently found in endocrine tumors (29\%) followed of ampullary tumors $(13 \% ; \mathrm{p}<0,01)$. No significant correlation was observed between the presence of a somatic EGFR mutation and clinicopathological variables. Fluorescence in situ hybridization (FISH) analysis did not demonstrate amplification in any tumors. Only three somatic mutations in the EGFR gene were detected in pancreatic ductal adenocarcinoma and no association was observed with the clinical variables. Our results suggest that EGFR mutations are rare in pancreatic tumors and not associated with clinical prognosis, and treatment response.
\end{abstract}

Correspondence to: Dr Antonio Lozano-Leon, Department of Gastroenterology and Foundation for Research in Digestive Diseases, University Hospital Santiago de Compostela, c/ Choupana s/n. Santiago de Compostela, Spain

E-mail: antoniolozan@gmail.com

Key words: epidermal growth factor receptor, fluorescence in situ hybridization, immunohistochemistry, pancreatic cancer, tissue micro array

\section{Introduction}

Interest in epidermal growth factor receptor (EGFR) has been heightened in light of the development of EGFR tyrosine kinase (TK) inhibitors, i.e., gefitinib ${ }^{\circledR}$ and erlotinib ${ }^{\circledR}(1)$. Systematic analysis of kinase genes has identified somatic mutations in the serine-threonine kinase gene in different cancers including pancreatic cancer. However, since not all patients exhibit the same response, there is considerable interest in prognostic indicators that might predict the response of chemotherapy treatment. Several author had reported that the presence of somatic alterations in the EGFR gene in nonsmall-cell lung cancer (NSCLC) tumors could predict clinical response to gefitinib-sensitive and refractory tumors, also has reported no relationship between EGFR expression levels and tumor sensitivity of this chemotherapy treatment (2).

EGFR is a $170-\mathrm{kD}$ TK receptor that dimerizes and phosphorylates several tyrosine residues upon binding of specific ligands including epidermal growth factor and transforming growth factor $\alpha$. These phosphorylated tyrosines serve as the binding sites for several signal transducers that initiate multiple signaling pathways resulting in cell proliferation, migration and metastasis, evasion from apoptosis, or angiogenesis, all of which are associated with cancer phenotypes. Downstream pathways include ras-raf-MEK-ERK, phosphatidylinositol-3 kinase-Akt and PAK-JNKK-JNK (3).

To date, most of the EGFR somatic mutations are reported in lung adenocarcinoma (including bronchioloalveolar carcinomas). The mutations have been detected in exons 18 , 19 and 21 which encode the intracellular kinase domain. Mutations detected in exon 18 alter the amino acid G719 in the P-loop, while those detected in exon 21 change amino acids in the activation domain (L858 and L861). The mutation observed in exon 19 is an in frame deletion that may alter the structure of $\alpha \mathrm{C}$ helix (4).

In pancreatic tumors, EGFR somatic mutations in the majority of the cases have not been detected. This affirmation is according to Immervoll et al (5) who reported a molecular analysis of the EGFR-RAS-RAF pathway in 51 pancreatic ductal adenocarcinoma but no mutations of were observed in EGFR gene. According to the mutational status 
Table I. Pathological and clinical information of the study group.

Characteristics

Age (years)
Mean
Range

Gender (n)

Male

Female

$29(56 \%)$

$23(44 \%)$

Stage

I

II

$14(27 \%)$

$15(29 \%)$

$7(13 \%)$

IV

Type

Adenocarcinoma

$34(65 \%)$

Ampullary carcinoma

$8(15 \%)$

7 (13)

Endocrine tumor

Anaplastic carcinoma

$1(2 \%)$

$1(2 \%)$

Mucinous carcinoma

Squamous carcinoma

$1(2 \%)$

Tumor size, pathologic

Median, $\mathrm{cm}$

3.4

Range, $\mathrm{cm}$

1-11

Chemotherapy

Yes

$18(35 \%)$

No

Differentiation grade

Well

$37(71 \%)$

Moderate

$11(21 \%)$

Poor

Perineural infiltration

Yes

$13(25 \%)$

No

$39(75 \%)$

Vascular permeation

Yes

$11(21 \%)$

No

$41(78 \%)$

Metastasis

Yes

No

$46(88 \%)$

of this gene in lung cancer and the availability of the current therapy for this; it is necessary to evaluate the principal mutations or polymorphisms in pancreatic cancer with the intention to extend and improve the quality of life of the patients.

In this study, we screened genetic variations in exons 19 and 21 of EGFR gene by nested PCR sequencing because in
Table II. Genetic variations in EGFR gene in pancreatic tumors.

\begin{tabular}{lclll} 
Patient & Exon & Mutation status & Nucleotide & Amino acid \\
\hline 01B11559 & 21 & Heterozygous & ACC $>$ ACT & R831C \\
02B01540 & 21 & Heterozygous & AGG $>$ AGA & R841R \\
03B2384 & 19 & Heterozygous & ACA $>$ ACG & T751T \\
\hline
\end{tabular}

$\mathrm{K}$, lysine; R, arginine; C, cysteine; $\mathrm{Y}$, tyrosine; A, alanine; $\mathrm{T}$, threonine; H: hystidine; E, glutamic acid; G, Glycine; S, serine; V, valine; I, isoleucine; L, leucine.

this region several other types of cancer have been reported with a great number of mutations. To further investigate the status of EGFR in pancreatic tumors we additionally conducted immunohistochemistry and FISH analysis in tumor and normal pancreatic tissue. Subsequently, the relationship between tumor EGFR status and clinicopathological variables was examined.

\section{Material and methods}

Patients and tissue samples. Fifty-two pancreatic tumors diagnosed between 1999 and 2004 were retrieved from the files of the University Hospital of Santiago de Compostela. Information on age, gender, stage, size, differentiation grade, perineural infiltration, vascular permeation metastasis, chemotherapy treatment was obtain by review of medical records. Different types of pancreatic tumor were included, the majority of the tumors were adenocarcinomas (see Table I). Tumor stage and grade are based on the guidelines of the American Joint Committee on Cancer (6).

All cases were histologically reviewed and the most representative tumor areas were selected; six samples of nonneoplastic pancreatic tissue were included as controls. A Tissue Arrayer (Beecher Instruments, Sun Prairie, WI, USA) was used to construct two different tissue microarray (TMA) blocks, according to conventional protocols. The representative areas of the tumor were marked in the paraffin blocks and a 1'5-mm-diameter cylinder from this area was transferred to a new recipient TMA block. Sections $4 \mu \mathrm{m}$ thick were cut from both TMA blocks.

Mutation analysis of EGFR. Genomic DNA was extracted from normal and tumor tissue using the Puregene Kit (Gemtra Systems ${ }^{\circledR}$ ) according to recommendations from the manufacturer. Samples were screened for mutations in exons 19 and 21. We focused on these two exons because in other cancers these exons are the most frequently mutated (1,5,7-9). Using primers previously described by Lynch et al (7) a nested polymerase chain reaction (PCR) was performed. Subsequently, the PCR products were sequenced separately in sense and antisense directions using Beckman Coulter CEQ-8000. All sequences were screened for the presence of mutations manually as well as by the Chromas software, and confirmed by two independent PCR amplifications. 
A

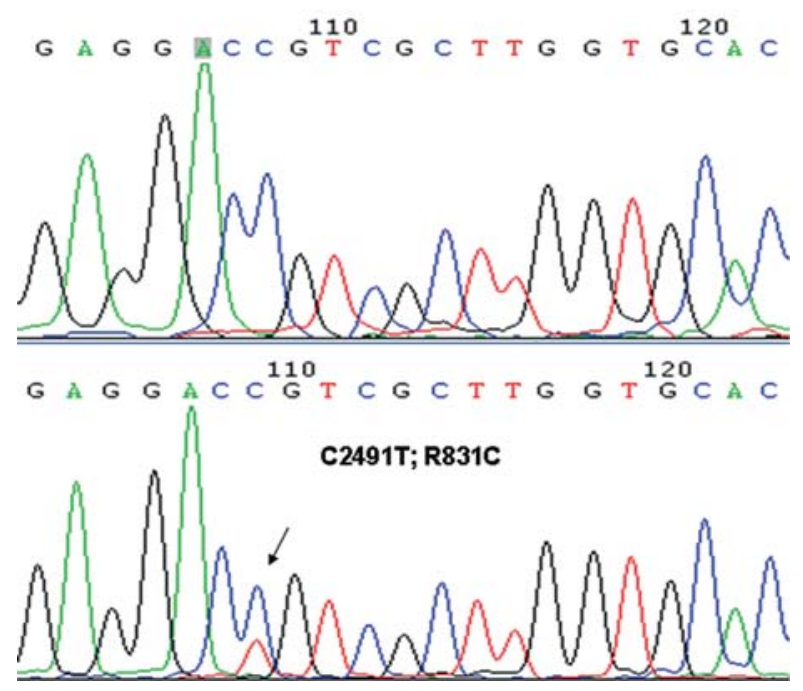

B
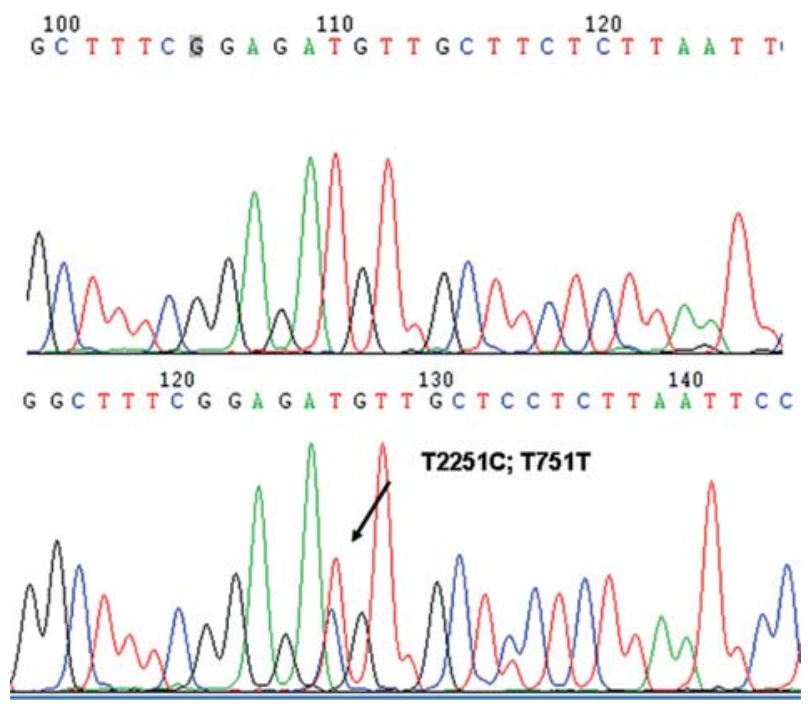

$\mathrm{C}$
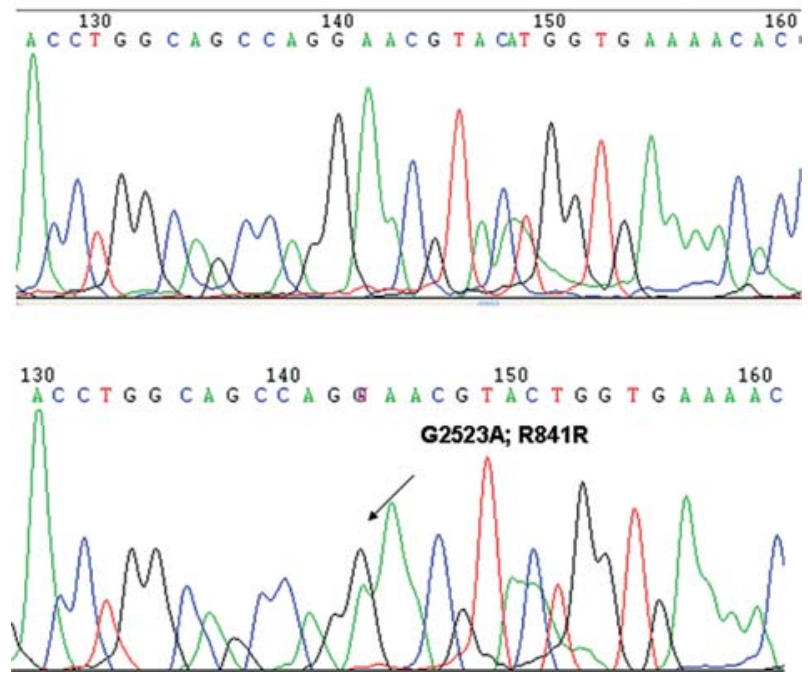

Figure 1. DNA sequence electropherograms illustrating the principal types of TK domain mutations and polymorphisms detected in EGFR gene. Each panel consist of tumor tissue (lower) and corresponding non-malignant pancreas tissue (upper) (A). Heterozygous somatic mutation in exon 21 of the EGFR gene C2491T; R831C (B). Heterozygous polymorphisms in exon 19, T2251C; T751T (C). Heterozygous polymorphisms in exon 21, G2523A; R841R.
EGFR expression. EGFR expression was assessed by immunohistochemistry (IHC) using a monoclonal antibody with the Dako EGFR PharmDx ${ }^{\mathrm{TM}}$ Kit (Dako Carpinteria, CA, USA), according to the manufacturer's instructions in an automated immunostainer (TechMate ${ }^{\mathrm{TM}} 500$ plus, Dako). Membrane staining intensity was evaluated using the $0,1+, 2+$ and $3+$ scales. Scores 0 were considered negative for EGFR expression, scores $1+$ weakly positive, $2+$ moderately positive and scores $3+$ strongly positive. Tumors were subsequently categorized as negative in 0 (no membranous and weakly staining) and 1 (strongly positive ( $\geq 50 \% 2+$ or $\geq 30 \% 3+$ ).

Fluorescence in situ hybridization. EGFR gene status was determined by FISH using EGFR/CEN7 probes mixture from Dako (Glostrup, Denmark) in the TMA used to determine EGFR expression. The technique was carried out according to manufacturer's recommendations. FISH signals were measured on a fluorescence microscope Nikon Eclipse (Japan) and digital images were taken with the Olympus DP-70 digital camera. FISH-positive control was used colon cancer cytology. A FISH-positive result was defined as presence of high polysomy ( $>4 E G F R$ gene copies per nucleus).

Statistical analysis. Statistical analysis was performed using SPSS v.15.0 software (SPSS Inc., Chicago, IL, USA). Associations between EGFR expression and individual clinicopathological variables were evaluated by the Chisquare test and the Pearson's correlation test. Survival time was defined as the time from resection surgery until death or last contact. The influence of independent binary variables on the incidence of EGFR overexpression was calculated by logistic regression modelling technique. $\mathrm{P}$-values $<0.05$ were considered statistically significant.

\section{Results}

Patient characteristics. Table I summarizes the clinical and histopathological characteristics of the study population. Twenty-nine $(56 \%)$ of the participant were male and the mean age was 64 years. Most of the tumors included in the study were adenocarcinomas.

Mutation profiles in pancreatic tumor. Genetic variations were found in three different pancreatic tumor samples (Table II). No mutations were detected in the six non-malignant pancreatic tissue samples. EGFR alterations were observed only in ductal pancreatic adenocarcinomas. Two alterations were observed in exon 21, one in exon 19. A heterozygous missense mutation was found in exon 21 (ACC-to-ACT; Arg-to-Cis) at codon 831 (R831C), in an adenocarcinoma from a male non-smoker with no response to chemotherapy treatment (gemcitabine $+5 \mathrm{FU}$ ). The patient developed metastases and local recurrence 6 months after the initial surgery. The other mutation in exon 21 (AGG-to-AGA; Arg-to-Arg) at codon 841 (R841R) was detected in a tumor from a male smoker, with no response to chemotherapy treatment (gemcitabine + $5 \mathrm{FU}$ ) and 731 days survival. The third mutation was found in exon 19 (ACA-to-ACG, Thr-to-Thr) at codon 571 ( $\mathrm{T} 571 \mathrm{~T})$, in a male smoker, with partial response to chemotherapy (reductions of size of tumor in $20 \%$ starting 

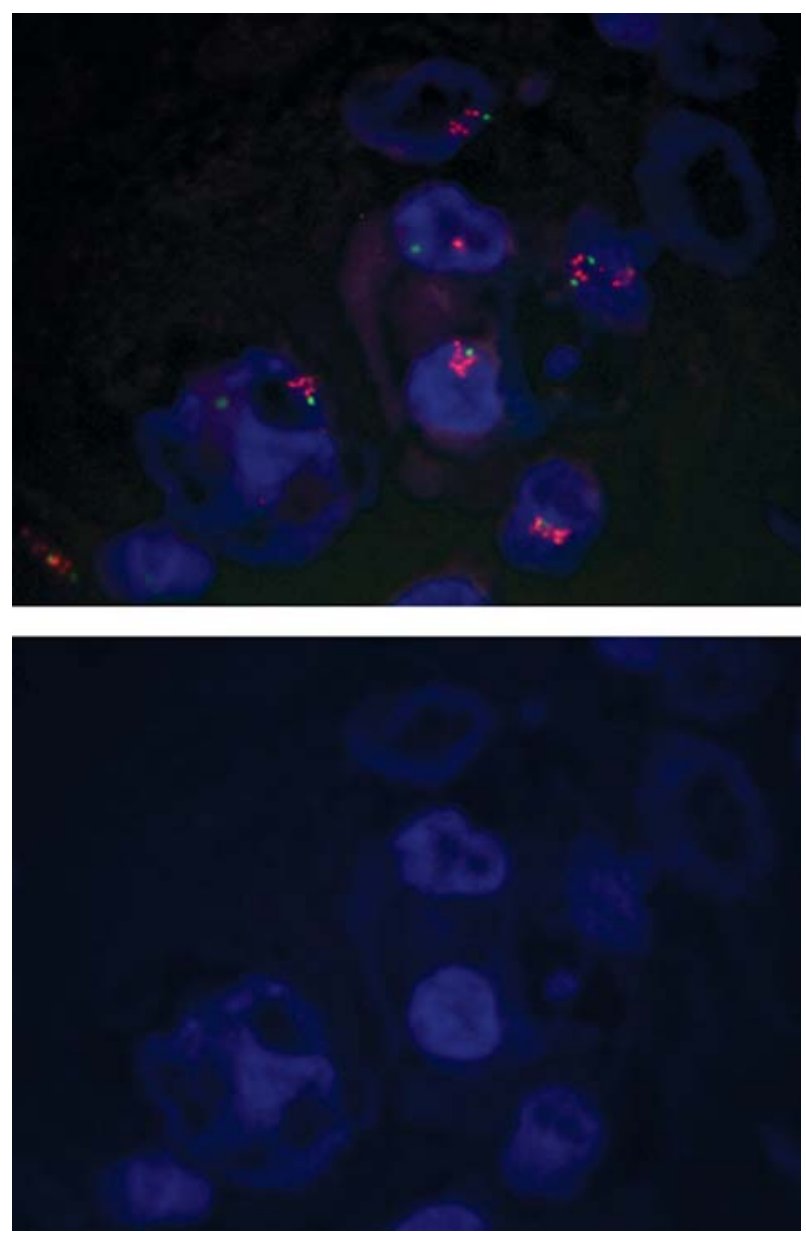

Figure 2. FISH analysis in pancreatic cancer tissue. (A) Control positive, amplification of EGFR gene in colon cancer. (B) No amplification of EGFR gene in tissue sample of pancreas cancer.

from the measure of the basal) and 1100 days of survival (Fig. 1).

EGFR expression. Four tumors (8\%) were strongly staining EGFR, 6 (12\%) were moderate overexpressed and 19 (37\%) were weakly positive. The overexpression $(3+)$ was more frequent in endocrine tumors $(29 \%)$ followed by ampullary carcinomas (13\%) (Fig. 2).

Correlation between genetic variation and expression of EGFR. No correlation was found between mutation and expression of EGFR. In tissue sample where the mutation was detected it showed only weakly positive expression. The effects of mutations in some clinical factors were examined but no significant correlation was observed. In a multivariate logistic regression analysis adjusted for sex, age, smoking, histology, stage and survival (days after surgery) in EGFR immunostaining (Fig. 3).

Fluorescence in situ hybridization. EGFR did not demonstrate amplification by fluorescence in situ hybridization in any of the samples studied.

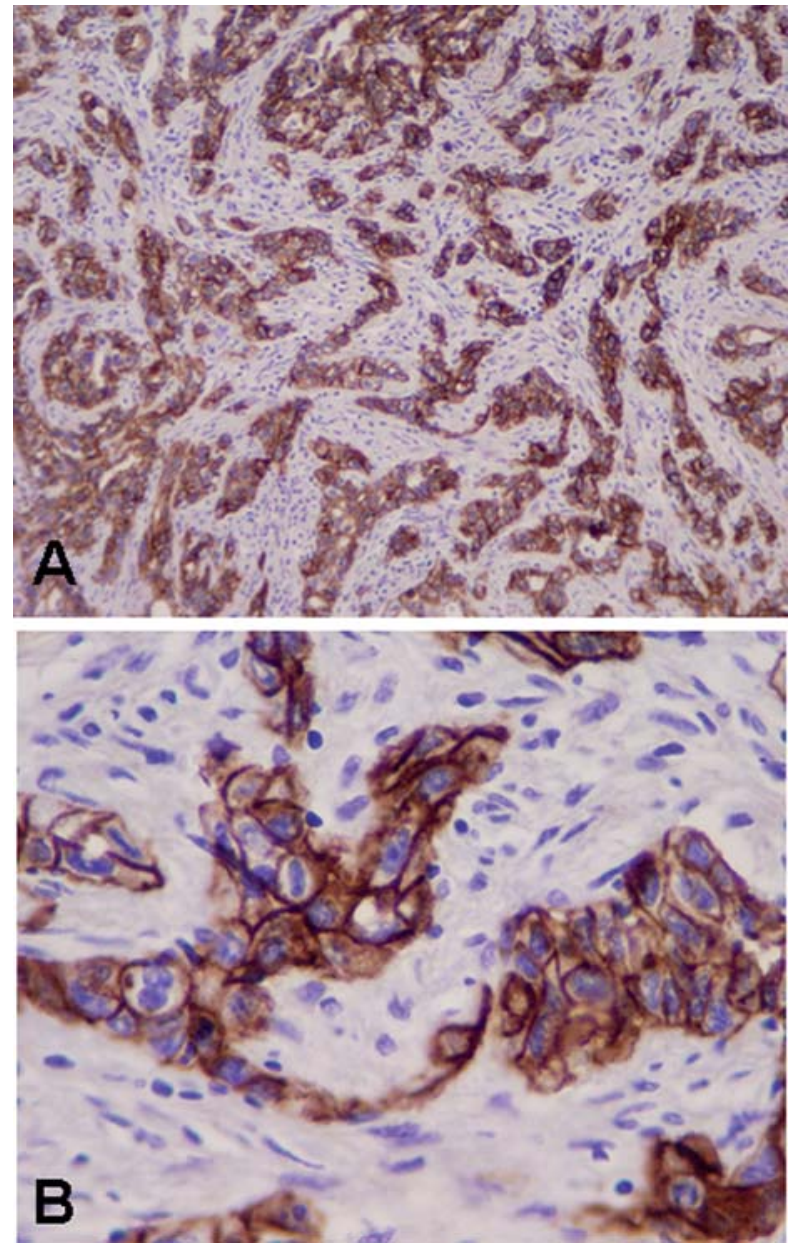

Figure 3. Representative findings on immunostaining for EGFR. (A) Positive immunostaining in pancreatic cancer tissue (x10). (B) Magnifications x40.

\section{Discussion}

This study was performed to assess the relationship between genetic variations and immunohistochemistry EGFR patterns with the clinical factors in pancreatic cancer. EGFR alterations were observed only in pancreatic ductal adenocarcinomas. When we compared mutation status with expression, EGFR overexpression was not correlated with the presence of a mutation in EGFR. A previous report (2) found significant overexpression of EGFR in cases where EGFR mutation was positive for lung cancer; their results suggest that one of the major contributors to EGFR overexpression is the TK domain mutation, although the exact mechanisms remain unclear. Three mutations in the EGFR gene were reported (8) in a study in non-small cell lung cancer (NSCLC). The three tumors samples had the same EGFR mutation, predicted to change leucine-858 to arginine (CTG-to-CGG; L858R). In pancreatic cancer EGFR overexpression occurs in $30-50 \%$ of adenocarcinomas, overexpression is associated with rapidly progressive disease, resistance to chemotherapy, and poor prognosis (5); However, in our study the immunohistochemical only showed overexpression in $4(8 \%)$ of the 52 tumors analyzed, two of them endocrine type $(9,10,11)$. We 
did not find correlations between the overexpresion and the clinical manifestations.

Several studies have shown EGFR associated with poor prognosis (12-14), whereas other trials have shown no prognostic association $(15,16)$. In concordance with Suzuki et al (2), the disparity in reports of the EGFR as a prognostic factor may reflect differences in detection methods, reagents, assay, cut-off points and population characteristics.

Three genetic variants in EGFR are reported in this study for exons 19 and 21 (Fig. 1). One of the somatic alterations (from arginine to cysteine at position 831) (R831C) was detected by Hannah et al (17) in patients with osteosarcoma. This mutation has not been previously described in other tumors. Because of the novelty of this finding, we confirmed this observation in 2 independent PCRs and sequencing reactions. These reactions were either performed on tumor material or a whole paraffin-embedded tissue sections with high tumor content. In the 3-dimensional model of the kinase domain, position 831 is adjacent to the C-terminal end of the important $\alpha \mathrm{c}$ helix. In protein kinase domains, this helix adopts positions characteristic of (auto) inhibited and activated states. In EGFR, most of the reported mutations found in NSCLC occur in or near this position. Whether the R831C mutation as a non-conservative change influences transmission of an activation signal, the phosphotransfer activity of the kinase domain, or is relevant to docking of adaptor molecules is presently unknown. Studies to determine the implications of these changes for EGFR activation and sensivity to EGFRtargeted compounds are currently ongoing.

We believe that oncogenic effects between mutated EGFR and adenocarcinomas may be consequence of deregulations in the principal pathway. The major downstream pathway mediating oncogenic effects of EGFR are in activation of ERKs via RAS (5), and ATK via $\mathrm{PI}_{3} \mathrm{~K}$ (18). Given the observation that EGFR mutants in the kinase domain selectively activated AKT pathway with no effects on the ERK pathway via RAS (19) the EGFR mutation would result in an increased survival of the effects of cancer cells by AKT. Although RAS mutants mainly activate the ERK pathway (20), they also activate AKT via $\mathrm{PI}_{3} \mathrm{~K}$ (21). Therefore, the K-RAS mutants would result in both increased survival and proliferation of the affected cancer cells. However, the exact consequences of the EGFR mutations in the complicated cellular contexts should be further analyzed (4).

FISH probes have been used by several groups in routine diagnostic of different types of cancer. Sauer et al (22) investigated the amplification of EGFR gene in colorectal carcinoma by fluorescence in situ hybridization. They found a $77 \%$ (37 cases of 48 ) positivity and they proposed that FISH probe might be a surrogate marker for EGFR mutation and that it can be used in a routine setting in pathology departments.

Other studies showed the effectiveness of the FISH probe in lung cancer for EGFR determination; Lynch (personal communication) detected 17 samples FISH positive out of 23 with mutation examined. Our study results indicate no amplification for EGFR gene.

Although we were able to demonstrate EGFR protein expression in a number of pancreatic tumors specimen, our mutational analysis on these limited number of clinical samples suggests that EGFR mutations are not a common event in pancreatic cancer. However, given the overall poor clinical prognosis of this tumor and the potential therapy implications of EGFR mutations, it would be important to extend our analysis to larger series of pancreatic cancer (ductal adenocarcinoma) and functionally characterize the mutation identified in this report.

In conclusion, three genetic variations were found in ductal pancreatic adenocarcinoma. Correlations between mutation or overexpression of EGFR gene and clinicopathological data were not found. The association among EGFR mutation status, clinical prognosis and response to anti-EGFR therapy described in NSCLC or other types of cancer may not be applicable to pancreatic cancer. However, it is utilized in combination with the standard procedure (gemcitabine), because the response to the treatment of antiEGFR does not necessarily correlate with EGFR expression.

\section{References}

1. Suzuki M, Shigematsu H, Hiroshima K and Iizasa T: Epidermal growth factor receptor expression status in lung cancer correlates with its mutation. Hum Pathol 36: 1127-1134, 2005.

2. Arteaga CL: Overview of epidermal growth factor receptor biology and its role as a therapeutic target in human neoplasia. Semin Oncol 29: 3-9, 2002.

3. Giaccone G, Gonzalez-Larriba JL and van Oosterom AT: Combination therapy with gefitinib, an epidermal growth factor receptor tyrosine kinase inhibitor, gemcitabine and cisplatin in patients with advanced solid tumors. Ann Oncol 15: 831-838, 2004.

4. Soung YH, Lee JW, Kim SY and Seo SH: Mutational analysis of EGFR and K-RAS genes in lung adenocarcinomas. Virchows Arch 446: 483-488, 2005.

5. Immervoll H, Hoem D and Kugarajh K: Molecular analysis of the EGFR-RAS-RAF pathway in pancreatic ductal adenocarcinomas: lack of mutations in the BRAF and EGFR genes. Virchows Arch 448: 788-796, 2006.

6. American Joint Commitee on Cancer: Manual de Diagnóstico de Extensión del Cáncer. AJCC: pp482, 2002.

7. Lynch TJ, Bell DW and Sordella R: Activating mutations in the epidermal growth factor receptor underlying responsiveness of non-small-cell lung cancer to gefitinib. N Engl J Med 350: 2129-2139, 2004.

8. Paez JG, Janne PA and Lee JC: EGFR mutations in lung cancer: correlation with clinical response to gefitinib therapy. Science 304: 1458-1461, 2004.

9. Papouchado B, Erickson LA, Rohlinger AL and Hobday TJ: Epidermal growth factor receptor and activated epidermal growth factor receptor expression in gastrointestinal carcinoids and pancreatic endocrine carcinomas. Mod Pathol 18: 1329-1335, 2005.

10. Fjällskog M-LH, Lejonklou MH and Öberg KE: Expression of molecular targets for tyrosine kinase receptor antagonists in malignant endocrine pancreatic tumors. Clin Cancer Res 9: 1469-1473, 2003.

11. Srivastava A, Alexander J and Lomakin I: Immunohistochemical expression of transforming growth factor $\alpha$ and epidermal growth factor receptor in pancreatic endocrine tumors. Hum Pathol 32: 1184-1189, 2001.

12. Hilbe W, Dirnhofer S and Oberwasserlechner F: Immunohistochemical typing of non-small cell lung cancer on cryostat sections: correlation with clinical parameters and prognosis. J Clin Pathol 56: 736-741, 2003.

13. Brattstrom D, Wester K and Bergqvist M: HER-2, EGFR, COX-2 expression status correlated to microvessel density and survival in resected non-small cell lung cancer. Acta Oncol 43: 80-86, 2004.

14. Fontanini G, De Laurentiis M and Vignati S: Evaluation of epidermal growth factor-related growth factors and receptors and of neoangiogenesis in completely resected stage I-IIIA nonsmall-cell lung cancer: amphiregulin and microvessel count are independent prognostic indicators of survival. Clin Cancer Res 4: 241-249, 1998. 
15. Hirsch FR, Varella-Garcia M and Bunn PA Jr: Epidermal growth factor receptor in non-small-cell lung carcinomas: correlation between gene copy number and protein expression and impact on prognosis. J Clin Oncol 21: 3798-3807, 2003.

16. Brabender J, Danenberg KD and Metzger R: Epidermal growth factor receptor and HER2-neu mRNA expression in non-small cell lung cancer Is correlated with survival. Clin Cancer Res 7: 1850-1855, 2001.

17. Hannah WY, Koeppen H and Garcia R: Epidermal growth factor receptor in osteosarcoma: expression and mutational analysis. Hum Pathol 38: 1184-1191, 2007.

18. Kamata $\mathrm{T}$ and Feramisco JR: Epidermal growth factor stimulates guanine nucleotide binding activity and phosphorylation of ras oncogene proteins. Nature 310: 147-150, 1984.
19. Sordella R, Bell DW and Haber DA: Gefitinib-sensitizing EGFR mutations in lung cancer activate anti-apoptotic pathways. Science 305: 1163-1167, 2004.

20. Vojtek AB, Hollenberg SM and Cooper JA: Mammalian Ras interacts directly with the serine/threonine kinase Raf. Cell 74: 205-214, 1993

21. Rodriguez-Viciana P, Warne PH, Dhand R and Vanhaesebroeck B Phosphatidylinositol-3-OH kinase as a direct target of Ras. Nature 370: 527-532, 1994.

22. Sauer T, Guren MG and Noren T: Demonstration of EGFR gene copy loss in colorectal carcinomas by fluorescence in situ hybridization (FISH): a surrogate marker for sensitivity to specific anti-EGFR therapy? Histopathology 47: 560-564, 2005. 